\title{
Semantic Classification of Road Markings from Geometric Primitives
}

\author{
Paul Amayo, Tom Bruls, and Paul Newman
}

\begin{abstract}
The classification of semantically meaningful road markings in images is an important and safety critical task for autonomous and semi-autonomous vehicles. However, beyond simple lane markings, real-time detection and interpretation of road markings is challenging as images are subject to occlusions, partial observations, lighting changes and differing weather conditions. Additionally, there is high variation in the road markings between countries and regions, which makes interpretation difficult. In this work we present a three-fold approach to the semantic classification. Firstly, we employ a weakly supervised neural network to detect pixels belonging to road markings under different conditions. Subsequently, these pixels are classified into geometric primitives, from which we retrieve the semantic classes through a fast and parallel modelfitting algorithm that offers real-time performance. Unlike other methods in the literature that perform road marking classification independently, our proposed approach performs a joint classification leveraging the highly structured configurations that characterise urban traffic scenes. Consequently, we retrieve the underlying semantic classes under a variety of weather and lighting conditions as we demonstrate in our results.
\end{abstract}

\section{INTRODUCTION}

The safe operation and deployment of a robot is intrinsically tied to its understanding of the work-space it operates in. In the case of an autonomous vehicles this extends from the knowledge of its location and its surroundings to the allowable behaviour at that particular location. The latter is mainly encoded into painted markings on the road surface which guide vehicles into acceptable behaviour and serve as warning for different hazards.

Offline mapping services such as Google Maps, HERE maps, and OpenStreetMap nowadays attempt to include these kind of details to aid autonomous driving. However, these offline systems do not fully negate the need for autonomous vehicles to be able to directly detect and interpret road markings in real-time through their live sensors for several reasons (i.e. roads are constantly changing, increasing, or undergoing maintenance). These off-line methods cannot directly compensate for this, leading to safety concerns for autonomous operation especially as regions that receive less traffic are considered.

Therefore, we focus on scene understanding for autonomous vehicles from live perception. More specifically, we present a method for the classification of a collection of road markings (i.e. not just lane markings) from a frontfacing monocular camera. We operate on the premise that the majority of the painted road markings originate from simple geometric primitives (i.e. lines), even though their scale and rotation differ greatly due to the camera perspective. The

The authors are with the Oxford Robotics Institute, Dept. Engineering Science, University of Oxford, UK. \{pamayo, tombruls, pnewman\} arobots.ox.ac.uk
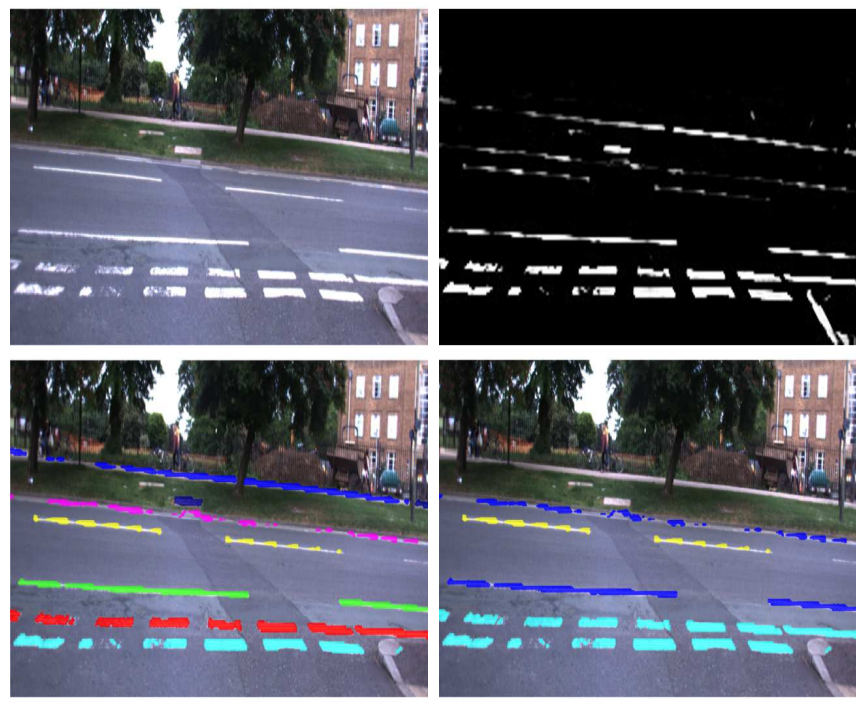

Fig. 1. Semantic classification of road markings in an urban environment as implemented in this paper. From an image taken by the front-facing camera of an autonomous vehicle (top left), pixels of potential road markings can be identified by a trained deep neural network (top right) under various lighting conditions. A fast and real-time two-step global energy optimisation approach then retrieves the road marking classes. The first optimisation step reveals geometric primitives (lines) which encode road marking segments (bottom left), before a further optimisation step classifies these primitives into semantically meaningful road markings as seen in the bottom right image.

spatial configuration of these primitives distinguishes the road marking classes.

Early approaches for road marking classification [1], [2] proposed the matching of features/shapes to obtain the road marking classes from geometric primitives, but generally struggle with changes in orientation and scale. Additionally, these approaches performed shape classification independently, neglecting the fact that combinations of road markings are often found in the same spatial configuration. Furthermore, there is a decrease of performances in scenes with occlusions or changes in lighting and weather conditions. End-to-end deep learning approaches [3] offer solutions to some of these problems. However, creating pixel-wise road marking classification labels is extremely labour expensive and cannot be done automatically as in [4]. Besides, no techniques currently exist for including domain knowledge (e.g. structure of the road scene or geometric primitives of road markings) directly into these deep learning frameworks.

In this paper, we propose an integrated framework for the detection and semantic classification of road markings. As demonstrated in Figure 1, pixels that belong to road markings are first identified by a trained deep neural network [4]. 
This allows for accurate detection of road marking pixels under varying lighting and weather conditions. From these pixels a two-step method retrieves the semantic road marking classes. This method is based on a fast, robust, real-time, global energy optimisation implemented through a COnvex Relaxation Algorithm (CORAL) [5]. Unlike most geometric multi-model fitting approaches, global energy approaches inherently consider the overall classification of data points to underlying models, in this case considering the spatial proximity of road marking classes. In the first optimisation step, geometric primitives (i.e. lines) from the identified pixels are extracted. These are then clustered through a subsequent optimisation, due to their specific configurations, to the different semantic classes through a further energy optimisation revealing a joint classification of road markings in a scene as shown in the bottom right of Figure 1. This removes the need for expensive and time-consuming manual annotations used for training purposes in proposed learning techniques [3], while still retaining strong and real-time classification performance.

In particular this work offers the following contributions:

- A robust, accurate method for extracting geometric primitives from road marking pixels.

- A fast, global labelling for the semantic classification of road markings using a combination of the geometric primitives and the road marking pixels.

- A method to track road marking classes from frame to frame.

The rest of the paper is organised as follows. In Section II related work in the area of road marking detection is summarised. In Section III the process for obtaining the road marking pixels is explained, followed by Section III$\mathrm{B}$ wherein the details of the geometric primitive extraction technique are described, before presenting the subsequent segmentation in Section III-C. In Section IV we introduce the road marking tracking pipeline before showing qualitative and quantitative results in Section V. Conclusions and discussion follow in Section VI.

\section{RELATED WORK}

Early work on the classification and interpretation of road markings was majorly concerned with the detection of lane markings, which form a subset of the road markings but are important for semi-autonomous vehicles as lanefollowing and lane departure warning systems are popular Advanced Driver Assistance Systems (ADAS). The survey by Hillel et al. [6] presents the common pipeline implemented by most lane marking detection algorithms using images. This pipeline includes a feature detection step to extract the outlines of the lane separators through edge or gradient detection, followed by a model fitting algorithm, usually a Hough transform for straight roads and a spline or polynomial model for curved roads. However, the feature detection is prone to occlusions and changes in illumination and weather conditions, while the model-fitting is dependent on correct parametrization and model choice. This limits the usability of these techniques to simple scenes (e.g. highway environments), not alike the urban environments studied in this paper.
To improve on the aforementioned problems, several approaches introduced an extra filtering step that attempts to extract regions of the viewed scene which contain road markings, as presented in the survey by Veit et al. [7]. While these are able to improve the baseline performance, they still suffer under varying illumination forcing the use of additional heuristics for practical functionality [8], [9]. Additionally as more complex road markings are sought the extracted features evolve from simple edges to retrieve lanes to more complex shape contours to detect arrows and spatial configurations for zebra crossings. Examples of these are descriptor-based approaches such as Histogram of Gradients (HOG) used in [10] and Fourier approaches used in [11], [12]. Classification of these features can then be performed by template matching as seen in [13], [14] or by heuristic shape-based rules. These techniques, while showing good performance on their evaluated datasets, do not generalise well and are sensitive to occlusions and partial observations limiting their practical use.

Supervised learning approaches offered a way to improve generalisation and thus several flavours of these appeared in the literature, ranging from KNN classification [15] and Support Vector Machines [16], [17] to shallow neural networks [18], [19]. It must be noted that these techniques each focus on a different specific subset of road markings, which are then independently detected and classified and hence generalisation to new features is limited. A divergence from this approach is presented by Bonolo et al. [20], who exploited the spatial relationship between road markings to improve classification using a Conditional Random Field (CRF). This however requires perfect detection of road markings and with the optimisation taking a few seconds per image it is not suited for online use.

More recently, deep networks have been successfully trained for road marking recognition [21], [22] or purely for classification [23]. However, these approaches either implement additional preprocessing algorithms or require detected road markings as an input, because of a lack of ground-truth road marking labels in large-scale urban datasets. The authors of [3] are the first to train a network on a large-scale (handlabelled) dataset and perform coarse road marking detection under challenging conditions.

In comparison to all of the aforementioned work, our approach leverages the power of deep learning to perform robust pixel-accurate road marking detection under difficult conditions and occlusions without the need for expensive road marking class labels, while still integrating domain knowledge to semantically classify road markings jointly in real time.

\section{SySTEM OVERVIEW}

In this work, we take a three-fold approach to the semantic classification of road markings. Firstly, we deploy a deep semantic segmentation network to detect road marking pixels $\mathbf{u} \in\left\{u_{1}, \cdots, u_{n_{p}}\right\}$, where $n_{p}$ is the number of detected pixels, in a monocular image. This non-trivial operation removes artefacts from the image such as cars, buildings, and other objects with line features, which introduce noise to the subsequent steps. 
Secondly, a global energy optimisation retrieves an $a$ priori unknown number of geometric primitives $A \in$ $\left\{A_{1}, \cdots, A_{n_{l}}\right\}$, where $n_{l}$ is the number of extracted primitives, from the road marking pixels which encode the road marking segments.

Lastly, we cluster these road marking segments into semantically meaningful classes $\psi \in\left\{\psi_{1}, \cdots, \psi_{n_{c}}\right\}$, with two types of constraints followed by a subsequent energy optimisation. This pipeline is described in more detail in the following subsections.

\section{A. Road Marking Detection}

We deploy a deep semantic segmentation network to identify image pixels that belong to the road markings. It can be seen [4] that in the presence of adequate training data, such a network will outperform existing techniques as it is able to exploit the global scene context, making it more robust to lighting changes, spatial deformations, degradation, and partial occlusions.

Creating adequate training data, in this case pixel-wise road marking labels, is extremely labour expensive. To bootstrap this, the monocular image is combined with a LiDAR reflectance point cloud to create road marking annotations in a weakly supervised way using several domain assumptions. We exploit the property that road markings are highly reflective and optimise a dense CRF over the image to detect the road marking pixels by relating them to highreflectance LiDAR points, which are not affected by lighting changes. This allows for the automatic generation of a large set of road marking annotations under various conditions, which are used for training purposes.

After the network is trained with these annotations, a road marking mask can be retrieved in real-time from a monocular image. Figure 2 shows that the network is robust to both appearance and lighting changes in the scene, providing a strong set of pixels from which individual road markings can be obtained. Due to space constraints we direct the reader to [4] for further details and the utilised network architecture.

\section{B. Road Marking Geometric Primitive Extraction}

After the identification of the road marking pixels by the trained deep segmentation network, we extract geometric primitives. linear segments, that encapsulate road marking segments. The number of road marking segments in a specific scene is a priori unknown, and apart from the trivial case of lane markings, they occur in different orientations and lengths with various levels of occlusion. Additionally, the network output also contains some level of noise, as objects of high reflectance (i.e. curbs) not corresponding to road markings can sometimes be wrongly segmented.

Several techniques for multi-line fitting in images such as vanishing point detection [3], RANSAC [24] and the Hough transform have been employed to obtain road marking segments. However, when moving away from the simple lane detection scenario performance of these techniques deteriorates. The Hough transform is highly dependent on parametrisation, while sequential RANSAC techniques struggle in scenes with noise and clutter, which cascades inaccuracy leading to poor outputs. In contrast, robust energy based

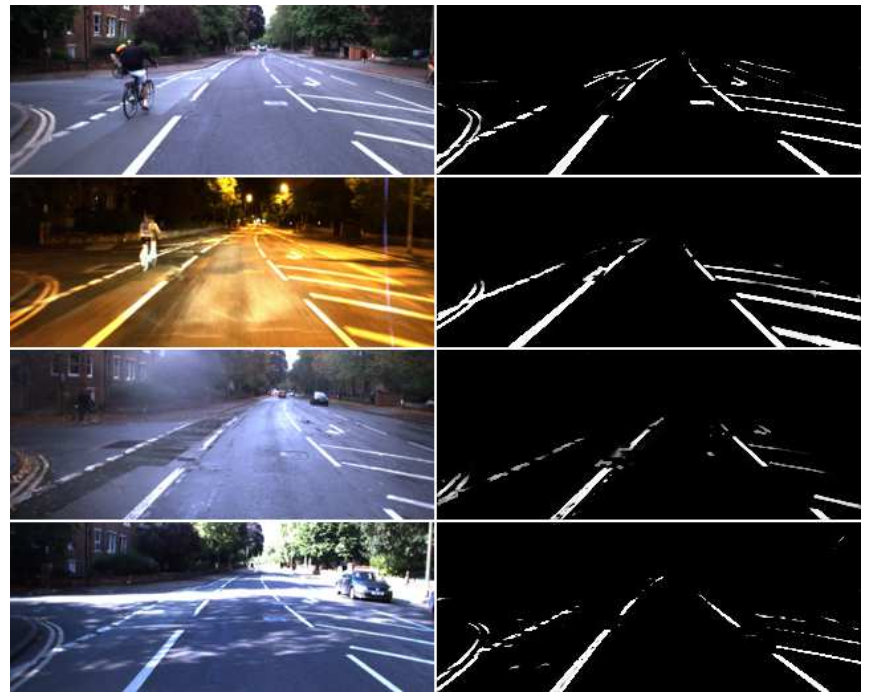

Fig. 2. Road marking detection under different lighting conditions (overcast, night, rainy, and sunny). Despite the large changes in the prevailing conditions the trained deep segmentation network [4] is able to accurately identify the pixels belonging to the road markings from the monocular image.

multi-model fitting approaches [25], [5] have been shown to outperform greedy approaches in the presence of noise. This is as energy based approaches take into consideration the overall classification of the data points to all the models. Firstly, by promoting locality through a smoothness prior that ensures that points that are close together have a similar model. In addition, these techniques are able to converge to the correct number of geometric models present in the data through a compactness prior.

With this in mind, we adopt the formulation given by CORAL [5] to perform the multi-line fitting. We define a global energy function:

$$
\begin{aligned}
& \underbrace{\sum_{l=1}^{n_{l}} \sum_{i=1}^{n_{r m}}\left(\left\|D\left(\mathbf{A}_{l}, \mathbf{u}_{i}\right)\right\|\right) \phi_{l}(\mathbf{u})}_{\text {Geometric Error Energy }}+\lambda \underbrace{\sum_{\text {Compactness Energy }}^{n_{l} \sum_{i=1}^{n_{r m}}\left|\nabla_{\mathcal{N}} \phi_{l}(\mathbf{u})\right|_{1}}}_{\text {Smoothness Energy }} \\
& +\underbrace{\beta\|L\|}_{i=1}
\end{aligned}
$$

The data term in Equation 1 accounts for the distance between a point and the geometric primitive. Here $A$ is the line equation $\mathbf{A}_{l}=\left(a_{l}, b_{l}, c_{l}\right)$ and we refer to $D$ as the Euclidean distance between a point $\mathbf{u}_{i}=\left(x_{i}, y_{i}\right)$ and the line $A_{l}$. Membership of data points to their respective model is encapsulated through the indicator function

$$
\phi_{l}(\mathbf{u})=\left\{\begin{array}{ll}
1 & \mathbf{u} \in A_{l} \\
0 & \text { otherwise }
\end{array},\right.
$$

which is self-constrained, $\sum_{l=1}^{n_{l}} \phi_{l}(\mathbf{u})=1$, such that a point can only be a member to one model. To account for outliers as not all data points might be explained by a linear segmenta special label $\emptyset$, representing the outlier model is added. In this way a constant cost $\gamma$ is assigned to points that cannot be explained by any geometric model. 

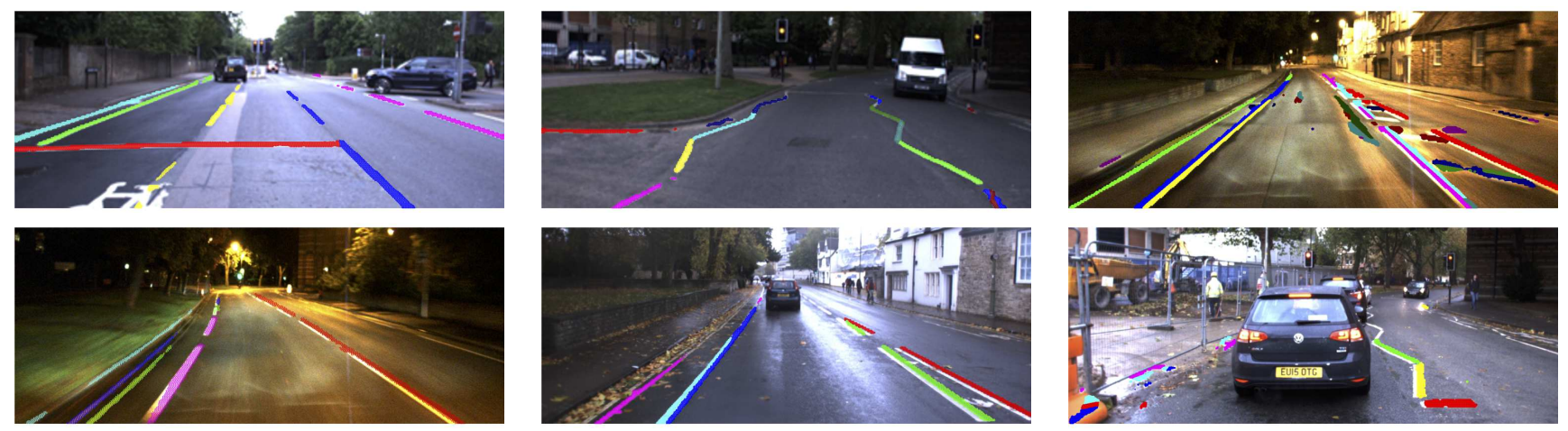

Fig. 3. A sample of the results from road marking geometric primitive extraction under different prevailing conditions. It can be seen that our proposed energy optimisation approach is able to accurately extract linear segments in a diversity of scenes. Revealing the underlying primitives for a large number of road markings present in the urban scene.

The smoothness term in Equation 1 promotes a homogeneous assignment of models to neighbouring points, introducing a spatial smoothness prior. By calculating the gradient $\nabla_{\mathcal{N}}$ of the indicator function over the neighbourhood $\mathcal{N}$ of a point given by its k-nearest neighbours, points that belong to the same neighbourhood but do not share the same model are penalised. The trade-off between the smoothness and data terms is controlled by the parameter $\lambda$. Finally, the compactness term in Equation 1 penalises the number of models by adding a constant cost $\beta$ per model. This penalises redundant models resulting in a more compact solution.

Minimisation of the energy in Equation 1 reveals the underlying geometric models. In CORAL a continuous optimisation approach leveraging a primal dual optimisation [26] is employed. This approach is inherently parallelisable allowing for easy implementation on General Purpose Graphical Processing Unit (GPGPU) hardware and real-time line model detection. Due to space constraints we refer the reader to [5] for further implementation details.

To reduce the search space for models in the CORAL optimisation, a finite number of models is usually proposed. In this work we use the Hough Transform for the model initialisation which generally proposes more models with low accuracy than are present in the scene. This is followed by an iterative process of primal dual optimisation for energy optimisation and model re-estimation up until the energy converges. Thus converging on $n_{l}$ road marking segments. A sample of the results can be seen in Figure 3, where this approach is able to extract the underlying primitives for a large number of road markings in a diversity of scenes.

\section{Road Marking Geometric Primitive Clustering}

The approach described in the previous section is able to accurately extract the road marking geometric primitives under different conditions. However, it is the underlying meaning encoded in these primitives that is actually interesting for autonomous driving. Therefore, we seek a clustering of the geometric primitives to perform classification. For the clustering, we utilise the idea that a collection of geometric primitives, however complicated, is still a geometric model albeit with more intra-class constraints $\theta(\cdot)$. Additionally, a set of fixed rules governs the spatial relationships between road marking classes which can be encoded into inter-class constraints $\Omega(\cdot)$.

Before the clustering can be performed, the effect of the camera perspective must be removed as it distorts the length, orientation and position of the road marking segments. By positioning a virtual camera above the observed scene this effect can be removed providing consistency not only in the detected road marking segments but also in their spatial configurations. This is performed through a homography warping of the scene, referred to as the Inverse Perspective Mapping (IPM) [20], which despite assuming that the road surface is planar works well in practice as seen in Figure 4.

We focus on the detection of six classes of road markings: single lane boundaries, double lane boundaries, lane separators, intersection markings, zig-zag, and junction road markings. Detection of these classes informs an autonomous vehicle about an upcoming road situation (e.g. a pedestrian crossing or a junction) or the allowable drivable area, both are crucial functions for autonomous driving.

Double lane boundaries, zig-zag and junction road markings all originate from a collection of geometric primitives and thus introduce intra-class constraints. These constraints are the angle and the distance between two road marking segments. Given the equation of a geometric primitive $\mathbf{A}_{l}=$ $\left(a_{l}, b_{l}, c_{l}\right)$, we introduce two simple constraints as follows

With these classes in mind two constraints were introduced, the angle between road marking segments and the corresponding distance between them. While simple these constraints encompass the possible configurations of the road marking classes and can be defined as:

$$
\begin{gathered}
\theta_{\text {angle }}\left(\mathbf{A}_{i}, \mathbf{A}_{j}\right)=\left|\arctan \left(a_{i} / b_{i}\right)-\arctan \left(a_{j} / b_{j}\right)\right| \\
\theta_{\text {dist }}\left(\mathbf{A}_{i}, \mathbf{A}_{j}, \mathbf{u}_{i}^{m}, \mathbf{u}_{j}^{m}\right)=\left(D\left(\mathbf{A}_{l}, \mathbf{u}_{i}^{m}\right)+D\left(\mathbf{A}_{l}, \mathbf{u}_{j}^{m}\right)\right) / 2,
\end{gathered}
$$

where $\mathbf{A}_{i}=\left(a_{i}, b_{i}, c_{i}\right)$ is the equation of the road marking segment $i$ and $\mathbf{u}_{i}^{m}$ is the midpoint of the pixels assigned to it.

By observing the classes it can be seen that the lane boundaries, lane separators and intersection road markings all consist of singular infinite line models. There is however, a strong prior that these classes appear parallel to each other allowing for the definition of an inter-class constraint that 

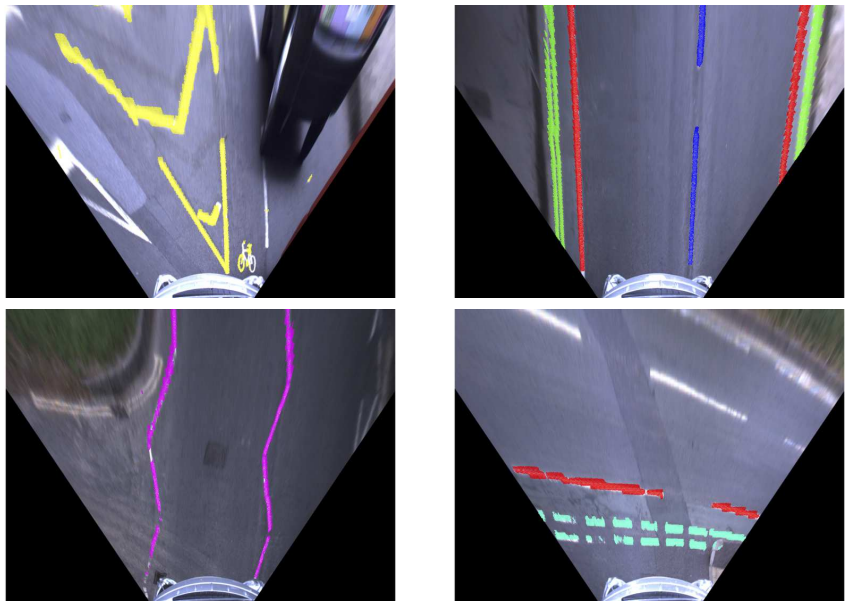

Fig. 4. Manually annotated road markings after inverse perspective mapping. These show a junction (top left), lane markings (top right), zigzag lines (bottom left), and a road intersection (bottom right) scene. As the semantic classes are encoded through a specific configuration of geometric primitives a subsequent CORAL optimisation reveals the underlying road marking classes.

penalises a collection of these if they are not parallel through the angle constraint. Similarly the double lane boundary consists of two parallel singular infinite line models within close proximity of each other, providing a inter-class constraints based on the angle and distance. Lastly, the angle is preserved in the zig-zag and junction crossings classes, producing a similar inter-class constraint.

We can thus propose several instances of these semantic classes through a targeted search that aims to find collection of lines that are parallel as well as those that fulfil the angle constraints of the zig-zag and junction classes. These instances form an initial set of "models" that are fed into the CORAL. The iterative optimisation of which reveals a compact set of semantic class instances as but additionally optimises for spatial smoothness, in essence performing a joint optimisation.

A sample of results of these is given in Figure 5, showing that this approach achieves high classification accuracy even in the presence of occlusions. To differentiate between the three classes of singular infinite line models in these results, the contiguity of their associated pixel inliers is used.

\section{ROAD MARKING TRACKING}

The previous section has described a framework for retrieving the road marking classes in a single image frame. Tracking of the road markings through consecutive frames improves the robustness of the classification, because, in most cases, road markings are seldom only seen in one frame, and persist from frame to frame. By exploiting this persistence our confidence of correct classification is increased when a road marking is detected over multiple frames. Additionally, some road markings are not fully observable in the current image (e.g. when traversing a road junction), making their classification ambiguous. By tracking classes, we can ensure that the correct assignment is made even when the road markings become partially observed.

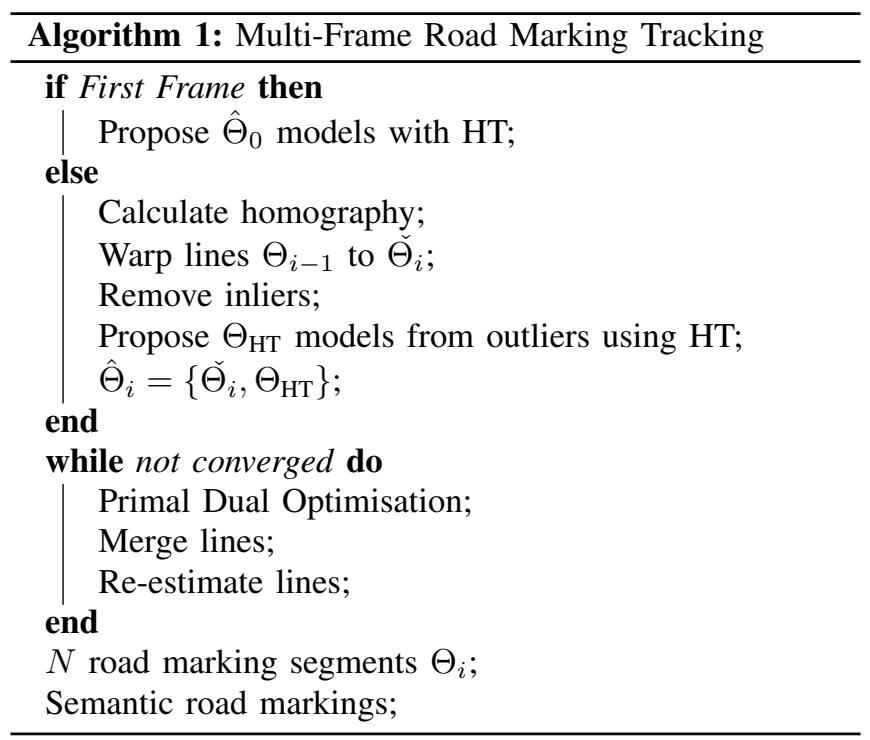

In this work, the selected road marking classes are collections of geometric primitives. These primitives can be tracked between subsequent frames if the motion $\mathbf{T}=\{\mathbf{R}, \mathbf{t}\}$ between the frames is known. We use the homography transformation between the subsequent frames to project a line model into the next frame

$$
\begin{aligned}
\mathbf{H} & =\mathbf{R}+\frac{\mathbf{t n}_{\text {plane }}^{t}}{d_{\text {plane }}} \\
\mathbf{A}_{i+1} & =\mathbf{H A}_{i} .
\end{aligned}
$$

These give an initial set of projected models for the subsequent frame, obtained from the initial frame. However, this set does not include all possible models, as road marking segments can appear for the first time in a particular frame. To cope with this, road marking pixels that are inliers to the projected models are first removed before a further Hough Transform (HT) initialisation is performed to the outliers availing new road marking geometric primitives as summarised in Algorithm 1. We implement a sliding-window approach to track road marking classes when they become fully occluded or the image becomes over-exposed. This allows detected semantic classes to persist further in time more accurately.

\section{EXPERIMENTAL RESUlTS}

To evaluate the presented approach, the Oxford RobotCar dataset [27] is used. This dataset consists of 100 repetitions of a $10-\mathrm{km}$ route in central Oxford under different prevailing weather and lighting conditions. Using the pre-trained deep segmentation network [4], we deploy our approach on three runs that were captured under vastly different conditions (overcast, rain, and night-time). It can be seen that our approach is able to retrieve the underlying semantic classes of the road markings in a variety of scenes even despite significant changes in appearance, as shown in Figure 5.

In Figure 6, we demonstrate the benefit of our road marking tracking algorithm. In this sequence, the junction markings become partially observable, leading to wrong 

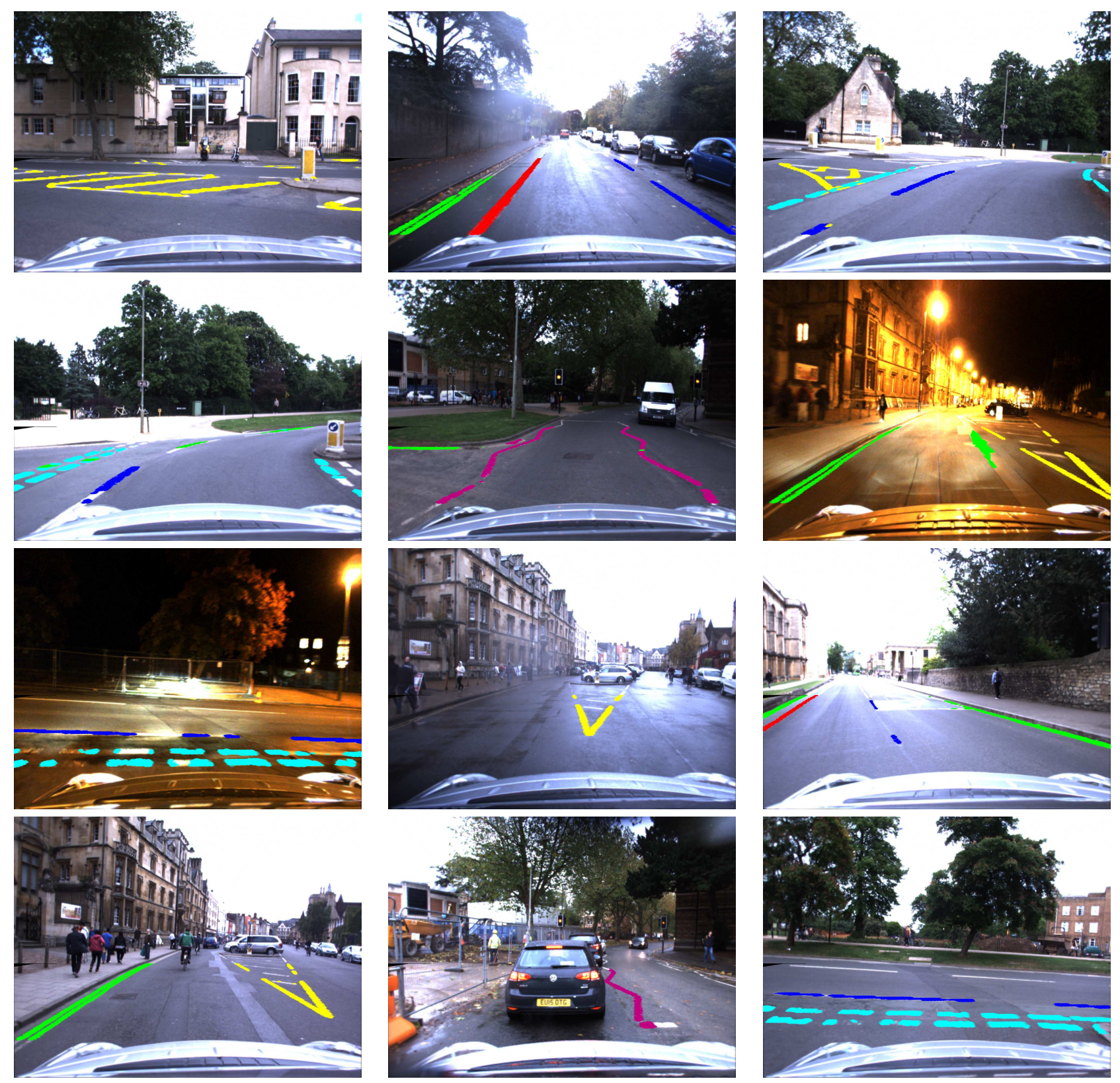

Fig. 5. Sample of results from the semantic classification of road marking pixels under different weather and lighting conditions (overcast/rain/night). By using a global energy approach, our proposed approach is able to detect multiple road marking segments in images from detected road marking pixels. These segments are aggregated through another energy minimisation into their semantic classes. Thereby, we reveal the underlying meaning of the road markings in complex urban environments, providing important cues for autonomous vehicles. These include indication for upcoming road situation, which could require specific behaviour. For instance, the zig-zag markers (purple) indicate an upcoming pedestrian crossing and the give-way dashes (cyan) indicate a junction.
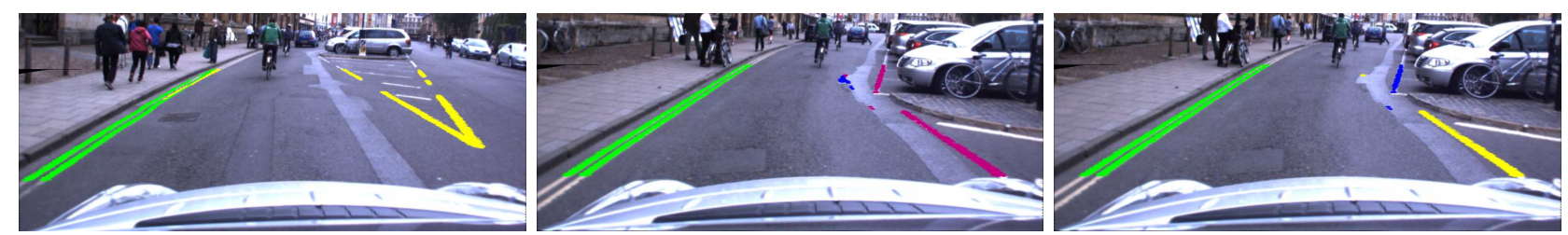

Fig. 6. Given the fully observed junction road marking (left), our approach is able to correctly detect the underlying semantic class. However, when the junction becomes partially observed (middle), the interaction of the underlying geometric primitives with others in the scene can cause mis-classification. In this case, the junction was labelled as a zig-zag line. By introducing road marking tracking (right), the correct class is retrieved even under partial observation, making the classification more robust. 
classification when only the current image frame is taken into account. By tracking the road markings, our approach memorises the scene and correctly initialises a new semantic road marking, increasing the robustness of the system.

The CORAL global energy optimisation is implemented using CUDA and deployed on an NVIDIA TITAN GPU. To obtain the running times, we averaged the computational time of 100 different images from the dataset. The timing results are presented in Table I. The results show that this method can be performed in real time $(\sim 6 \mathrm{~Hz})$, allowing online road marking classification.

\section{TABLE I}

Timing Results For the RoAD MARKing SEgmentation

\begin{tabular}{|l|r|}
\hline \multicolumn{1}{|c|}{ Module } & Time (ms) \\
\hline Road marking pixel detection & 16 \\
Energy minimisation (line models) & 110.8 \\
Energy minimisation (semantic classes) & 38.4 \\
\hline
\end{tabular}

\section{Conclusion}

In this paper we have presented a framework for the classification and interpretation of road markings in complex urban environments under varying weather conditions. From detected road marking pixels, this approach describes the semantic classes as different configurations of primitive geometric models and then employs a fast energy minimisation to extract the respective class in real time. By detecting certain classes we are able to reason about upcoming road situations, which could require specific behaviour. Unlike most of the contemporary approaches, we classify road markings jointly without requiring expensive manual annotations and are able to perform well in the presences of occlusions and degradation. Furthermore, the method is easily extendable to more classes and is thus able to provide an important cue for planning and navigation in urban scenes.

\section{ACKNOWLEDGEMENTS}

The authors acknowledge the following funding sources. Paul Amayo is funded by the Rhodes Trust. Paul Newman is supported by EPSRC Programme Grant EP/M019918/1.

\section{REFERENCES}

[1] G. Maier, S. Pangerl, and A. Schindler, "Real-time detection and classification of arrow markings using curve-based prototype fitting," in Intelligent Vehicles Symposium (IV), 2011 IEEE. IEEE, 2011, pp. 442-447.

[2] X. Du and K. K. Tan, "Comprehensive and practical vision system for self-driving vehicle lane-level localization," IEEE transactions on image processing, vol. 25, no. 5, pp. 2075-2088, 2016.

[3] S. Lee, J. Kim, J. S. Yoon, S. Shin, O. Bailo, N. Kim, T.-H. Lee, H. S. Hong, S.-H. Han, and I. S. Kweon, "Vpgnet: Vanishing point guided network for lane and road marking detection and recognition," in Computer Vision (ICCV), 2017 IEEE International Conference on. IEEE, 2017, pp. 1965-1973.

[4] T. Bruls, W. Maddern, A. A. Morye, and P. Newman, "Mark yourself: Road marking segmentation via weakly-supervised annotations from multimodal data," in Robotics and Automation (ICRA), 2018 IEEE International Conference on. IEEE, 2018, p. in press.

[5] P. Amayo, P. Piniés, L. M. Paz, and P. Newman, "Geometric MultiModel Fitting with a Convex Relaxation Algorithm," in Proceedings of the IEEE International Conference on Computer Vision and Pattern Recognition (CVPR), Salt Lake City, USA, June 2018.
[6] A. B. Hillel, R. Lerner, D. Levi, and G. Raz, "Recent progress in road and lane detection: a survey," Machine vision and applications, vol. 25, no. 3, pp. 727-745, 2014.

[7] T. Veit, J.-P. Tarel, P. Nicolle, and P. Charbonnier, "Evaluation of road marking feature extraction," in Intelligent Transportation Systems, 2008. ITSC 2008. 11th International IEEE Conference on. IEEE, 2008, pp. 174-181.

[8] R. Danescu and S. Nedevschi, "Detection and classification of painted road objects for intersection assistance applications," in Intelligent Transportation Systems (ITSC), 2010 13th International IEEE Conference on. IEEE, 2010, pp. 433-438.

[9] Z. Liu, S. Wang, and X. Ding, "Roi perspective transform based road marking detection and recognition," in Audio, Language and Image Processing (ICALIP), 2012 International Conference on. IEEE, 2012, pp. 841-846.

[10] N. Dalal and B. Triggs, "Histograms of oriented gradients for human detection," in Computer Vision and Pattern Recognition, 2005. CVPR 2005. IEEE Computer Society Conference on, vol. 1. IEEE, 2005, pp. 886-893.

[11] J. M. Collado, C. Hilario, A. de la Escalera, and J. M. Armingol, "Detection and classification of road lanes with a frequency analysis," in Intelligent Vehicles Symposium, 2005. Proceedings. IEEE. IEEE, 2005, pp. 78-83.

[12] J. M. Collado, C. Hilario, A. De La Escalera, and J. M. Armingol, "Adaptative road lanes detection and classification," in International Conference on Advanced Concepts for Intelligent Vision Systems. Springer, 2006, pp. 1151-1162.

[13] P. Foucher, Y. Sebsadji, J.-P. Tarel, P. Charbonnier, and P. Nicolle, "Detection and recognition of urban road markings using images," in Intelligent Transportation Systems (ITSC), 2011 14th International IEEE Conference on. IEEE, 2011, pp. 1747-1752.

[14] S. Vacek, C. Schimmel, and R. Dillmann, "Road-marking analysis for autonomous vehicle guidance." in EMCR, 2007.

[15] J. Rebut, A. Bensrhair, and G. Toulminet, "Image segmentation and pattern recognition for road marking analysis," in Industrial Electronics, 2004 IEEE International Symposium on, vol. 1. IEEE, 2004, pp. $727-732$.

[16] L. Gang, M. Zhang, L. Zhang, and J. Hu, "Automatic road marking recognition for intelligent vehicle systems application," Advances in Mechanical Engineering, vol. 9, no. 5, p. 1687814017706267, 2017.

[17] D. Ding, J. Yoo, J. Jekyo, S. Jin, and S. Kwon, "Efficient roadsign detection based on machine learning," Bulletin of Networking, Computing, Systems, and Software, vol. 4, no. 1, pp. 15-17, 2015.

[18] A. Kheyrollahi and T. P. Breckon, "Automatic real-time road marking recognition using a feature driven approach," Machine Vision and Applications, vol. 23, no. 1, pp. 123-133, 2012.

[19] J. Yamamoto, S. Karungaru, and K. Terada, "Road surface marking recognition using neural network," in System Integration (SII), 2014 IEEE/SICE International Symposium on. IEEE, 2014, pp. 484-489.

[20] B. Mathibela, P. Newman, and I. Posner, "Reading the road: Road marking classification and interpretation," IEEE Trans. Intelligent Transportation Systems, vol. 16, no. 4, pp. 2072-2081, 2015. [Online]. Available: http://dx.doi.org/10.1109/TITS.2015.2393715

[21] O. Bailo, S. Lee, F. Rameau, J. S. Yoon, and I. S. Kweon, "Robust road marking detection and recognition using density-based grouping and machine learning techniques," in Applications of Computer Vision (WACV), 2017 IEEE Winter Conference on. IEEE, 2017, pp. 760-768.

[22] T. Chen, Z. Chen, Q. Shi, and X. Huang, "Road marking detection and classification using machine learning algorithms," in Intelligent Vehicles Symposium (IV), 2015 IEEE. IEEE, 2015, pp. 617-621.

[23] T. Ahmad, D. Ilstrup, E. Emami, and G. Bebis, "Symbolic road marking recognition using convolutional neural networks," in Intelligent Vehicles Symposium (IV), 2017 IEEE. IEEE, 2017, pp. 1428-1433.

[24] M. A. Fischler and R. C. Bolles, "Random sample consensus: a paradigm for model fitting with applications to image analysis and automated cartography," Communications of the ACM, vol. 24, no. 6 , pp. 381-395, 1981 .

[25] H. Isack and Y. Boykov, "Energy-based geometric multi-model fitting," International journal of computer vision, vol. 97, no. 2, pp. 123-147, 2012.

[26] A. Chambolle and T. Pock, "A first-order primal-dual algorithm for convex problems with applications to imaging," Journal of Mathematical Imaging and Vision, vol. 40, no. 1, pp. 120-145, 2011.

[27] W. Maddern, G. Pascoe, C. Linegar, and P. Newman, "1 year, $1000 \mathrm{~km}$ : The oxford robotcar dataset," The International Journal of Robotics Research, vol. 36, no. 1, pp. 3-15, 2017. [Online]. Available: http://dx.doi.org/10.1177/0278364916679498 